\title{
Endovascular Intervention in Basilar Artery Entrapment within the Longitudinal Clivus Fracture: A Case Report
}

\author{
Fumiaki KANAMORI, ${ }^{1,2}$ Takashi YAMANOUCHI, ${ }^{1}$ Yuya KANO, ${ }^{3}$ \\ and Naoki KOKETSU ${ }^{1}$
}

\author{
${ }^{1}$ Department of Neurosurgery, Tosei General Hospital, Seto, Aichi, Japan; \\ ${ }^{2}$ Department of Neurosurgery, Nagoya University Graduate School of Medicine, \\ Nagoya, Aichi, Japan; \\ ${ }^{3}$ Department of Neurology, Tosei General Hospital, Seto, Aichi, Japan
}

\begin{abstract}
Although vascular complications after head trauma is well recognized, basilar artery entrapment within the longitudinal clivus fracture is rare. A 69-year-old man presented with progressive disturbance of consciousness and right hemiplegia after trauma. Computed tomography scan showed a right-sided acute subdural hematoma and multiple skull fractures, including a longitudinal clivus fracture. Magnetic resonance imaging revealed basilar artery occlusion and a small infarction at the ventral part of the pons. On the assumption of acute arterial occlusion caused by thrombus, endovascular thrombectomy was attempted, but resulted in perforation. After the procedure, basilar artery entrapment within the longitudinal clivus fracture turned out to be the cause of the occlusion. The present case suggests that basilar artery entrapment within the longitudinal clivus fracture is a possible cause of neurological deficits after trauma. In this subset, endovascular intervention without a correct diagnosis of this phenomenon is high risk.
\end{abstract}

Key words: basilar artery entrapment within the longitudinal clivus fracture, endovascular intervention

\section{Introduction}

Basilar artery entrapment within the longitudinal clivus fracture is a rare vascular complication after trauma. In this condition, basilar artery is sandwiched by the fractured clivus bones, resulting in arterial occlusion or stenosis. Despite the poor prognosis due to posterior circulation infarction, ${ }^{1)}$ the diagnosis is difficult in most cases and appropriate treatment has not been established.,2,3)

Here, we report a case of basilar artery entrapment within a longitudinal clivus fracture, for which we attempted endovascular intervention based on misdiagnosis.

\section{Case Report}

A 69-year-old man was transferred to our hospital due to a fall from stairs. On admission, the patient

Received October 6, 2017; Accepted January 9, 2018

Copyright $\odot 2018$ by The Japan Neurosurgical Society This work is licensed under a Creative Commons AttributionNonCommercial-NoDerivatives International License. presented with Glasgow Coma Scale (GCS) score of 13 and slight right hemiparesis. A computed tomography (CT) scan showed a right-sided acute subdural hematoma and frontal lobe contusion (Fig. 1A). The sphenoid sinus and the ethmoid sinus were filled with blood. On the bone window, multiple skull fractures were detected: a longitudinal clivus fracture extending to the sphenoid sinus, a right frontal bone fracture involving the skull base, a right temporal bone fracture, and a right sphenoid bone fracture at the lesser wing (Fig. 1B).

After 13 hours of conservative treatment, his consciousness showed a sharp decline to a GCS score of 5 , and his right hemiparesis got worse to complete hemiplegia. Brain MR imaging was performed immediately. Basilar artery was occluded at the proximal segment, and acute infarction on the left side of the ventral pons was detected (Fig. 1C). On the assumption of acute basilar artery occlusion caused by thrombus, endovascular intervention was performed to save the ischemic penumbra in the posterior circulation.

Right vertebral angiography showed basilar artery occlusion at the middle segment (Fig. 2A). Perfusion 

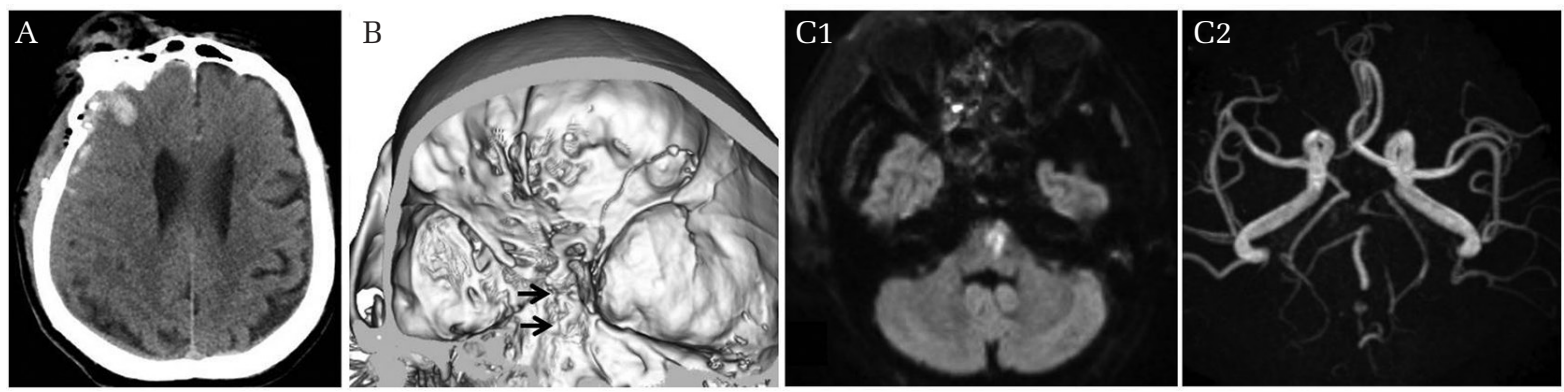

Fig. 1 (A) Computed tomography scan showing the right-sided acute subdural hematoma and the right frontal lobe contusion. (B) Three-dimensional reconstruction of the bone window of the computed tomography scan showing multiple skull base fractures, particularly a longitudinal clivus (arrow) fracture. (C) Magnetic resonance images. (1) Diffusion-weighted image showing the acute infarction in the left caudal pons. (2) Magnetic resonance angiogram showing the occlusion of the basilar artery.
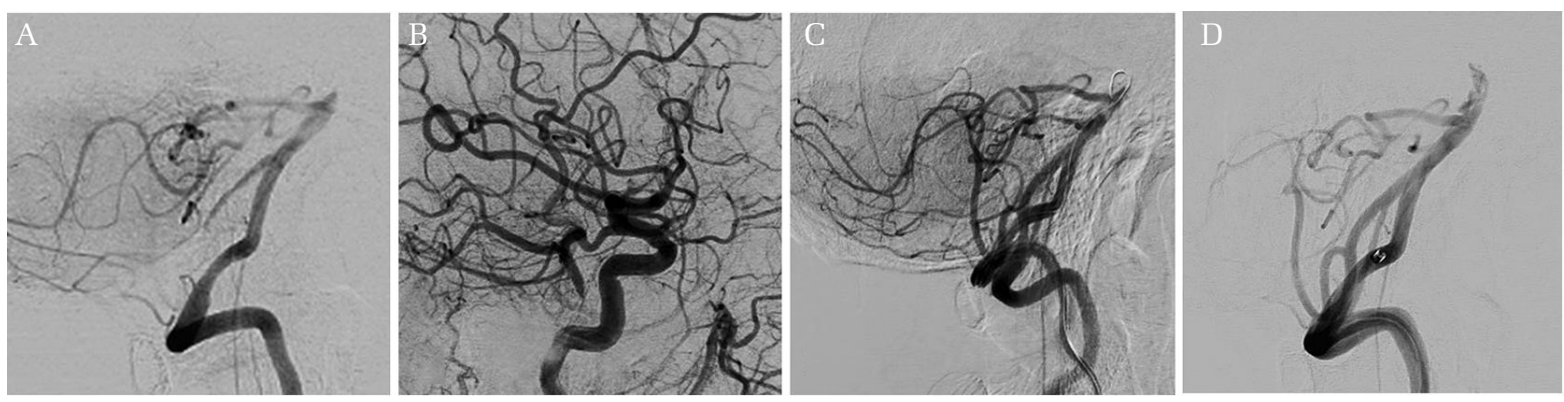

Fig. 2 Right vertebral angiogram demonstrating the occlusion of the basilar artery at the middle segment. (A) Right common carotid angiogram showed that the distal segment of the basilar artery was filled through the right posterior communicating artery. (B) Right vertebral angiogram after lesion crossing showing the deviation of the microguidewire from the basilar artery. (C) Right vertebral angiogram after the failure of lesion crossing showing the leakage of contrast agent from the basilar artery.

for distal basilar artery was preserved by collateral flows through posterior communicating artery (Fig. 2B). A direct-aspiration first-pass technique with a 5 French aspiration catheter (5Max Ace; Penumbra, Alameda, CA, USA) was attempted first, but could not catch the thrombus. Then, we tried to cross the occlusion site with a j-shaped microguidewire (Chikai 14; Asahi Intecc, Aichi), but the site was too hard to cross, resulting in perforation (Fig. 2C, 2D). The extravasation stopped spontaneously, and we finished the procedure at that point. A post-procedural CT angiogram revealed that the occluded part of basilar artery is trapped in the longitudinal clivus fracture (Fig. 3).

We did not initiate antiplatelet or anticoagulant therapy because of the intracranial hemorrhage and other traumatic complications. The patient's neurologic condition improved gradually to GCS score of 10, leaving severe right hemiparesis. About 6 months after this event, he is now under rehabilitation aiming for home life.

\section{Discussion}

Basilar artery entrapment within the longitudinal clivus fracture is a rare vascular complication after trauma. As subtypes, vertebral artery entrapment within the clivus and basilar artery herniation into the sphenoid sinus are known. Seventeen cases have been reported previously, and those outcomes are poor (Table 1). ${ }^{1-17)}$ Of these 17 patients, 7 died, 1 remained in a vegetative state, 3 suffered from locked-in syndrome, and 5 had some paralysis.

The mechanism of basilar artery entrapment within the longitudinal clivus fracture is proposed as follows: (1) the trauma, which causes arrest of forward motion of the skull, produces a longitudinal clivus fracture and lacerates the dura; (2) inertia of the brainstem and cerebellum, which continue to move forward relative to the skull, thrust the basilar artery into the breach of the longitudinal clivus fracture; and (3) the longitudinal fracture then closes while inserting basilar artery 

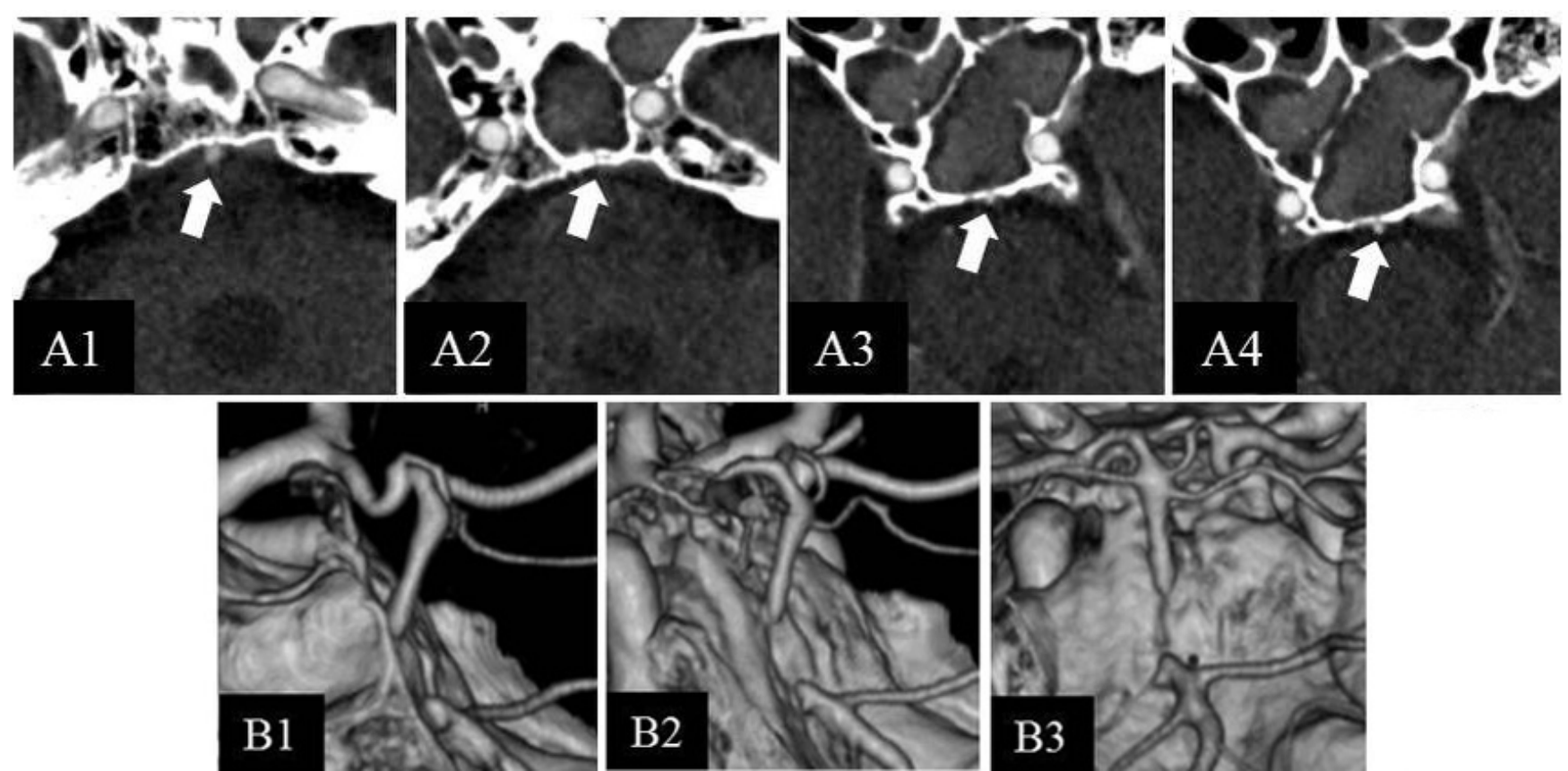

Fig. 3 (A) Axial images of computed tomographic angiography showing the basilar artery (arrow) disappearing superiorly and inferiorly into the line of longitudinal clivus fracture. (B) Three-dimensional reconstruction images of computed tomographic angiography: (1) lateral view, (2) oblique view, (3) anteroposterior view, showing the basilar artery entrapment within the longitudinal clivus fracture.

or perforators between bone fragments, resulting in arterial occlusion, stenosis, or dissection. ${ }^{6)}$ In addition to these direct mechanisms, subsequent thrombosis is another important factor. Injured intima and turbulent blood flow in stenosed or dissected artery lead to a formation of thrombus, which can narrow the arterial lumen even more and became a possible nidus for distal embolism. This subsequent event could account for some of the delayed deteriorations reported previously. ${ }^{1,3,5,11,15)}$

Diagnosis can be made by CT angiography, ${ }^{1)}$ which illustrates the exact image of basilar artery entrapment between clivus bone fragments. Without bone information and correct interpretation, the diagnosis is difficult because the clinical characteristics could mimic simple thromboembolic occlusion. This misdiagnosis can lead to high-risk endovascular intervention, as of the present case.

Effective treatment has not been established yet. Anti-thrombotic treatment is supposed to be effective for preventing aforementioned thrombotic progression. Four patients have received anti-coagulant or anti-platelet treatment. ${ }^{1,10,11,16)}$ Although none of these patients demonstrated deterioration after medication, its effect is unclear in this small number. In addition, anti-thrombotic treatment in trauma patients is sometimes risky. Although there is no such report so far, endovascular treatment could be usable for some specific conditions. ${ }^{18-21)}$ Endovascular aspiration could be effective for patients presenting deterioration due to subsequent thrombosis, ${ }^{1,5)}$ and stent placement might be under consideration for patients with basilar artery stenosis or dissection.

The present case suggests that basilar artery entrapment within the longitudinal clivus fracture could be a cause of neurological deficits after trauma. In this subset, endovascular intervention without a correct diagnosis is highly risky.

\section{Acknowledgments}

We would thank Dr. Mamoru Ishida for English language review.

\section{Conflicts of Interest Disclosure}

None of the authors have any conflicts of interest to disclose. 


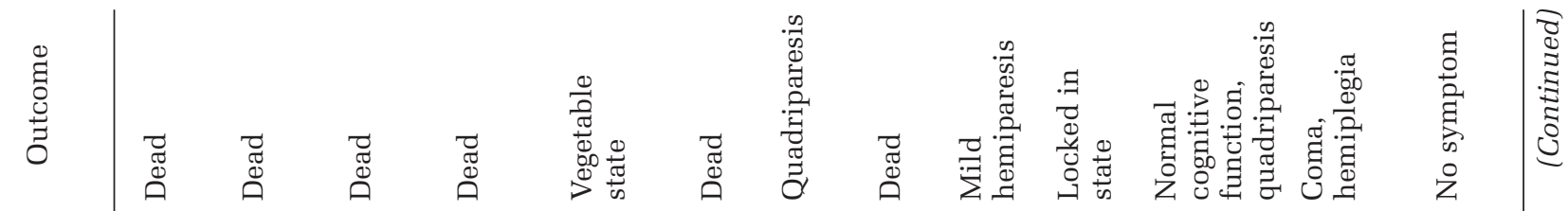

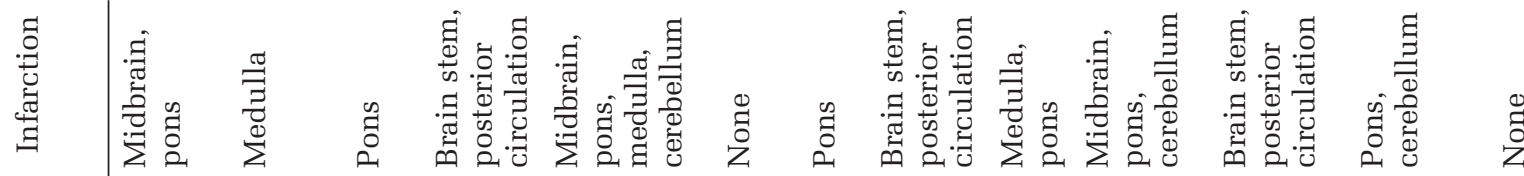

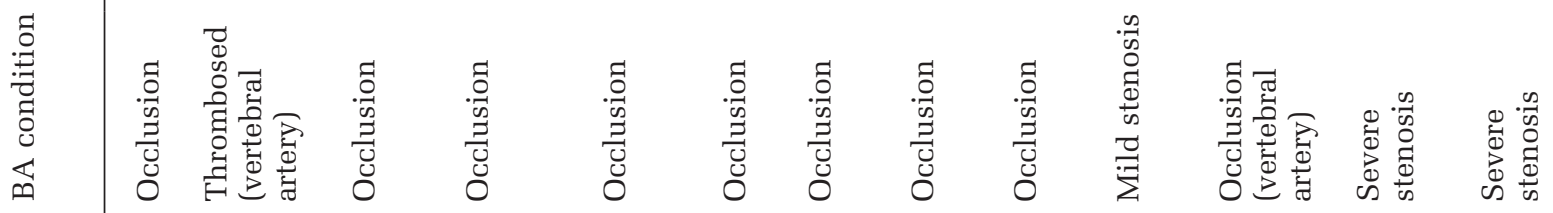

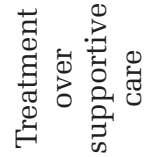

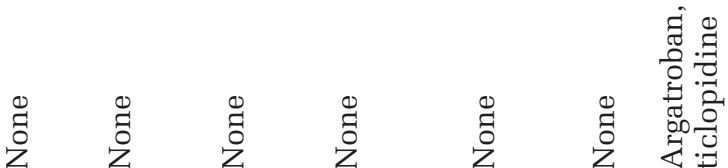
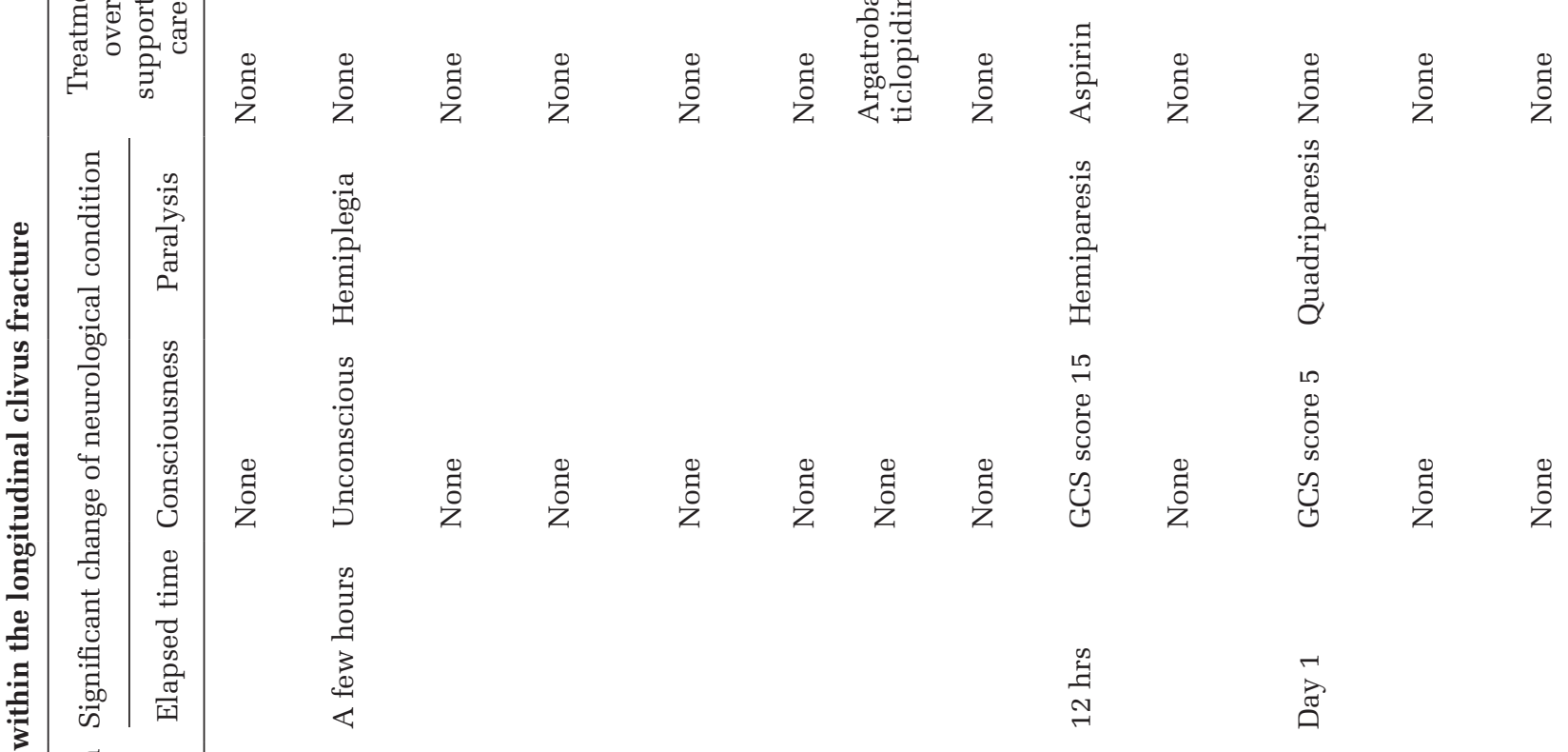

$\frac{20}{2}$
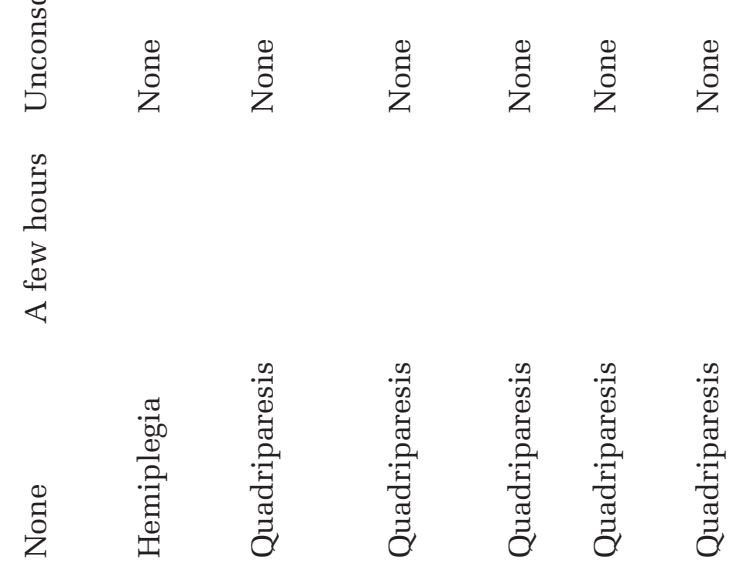

ํㅗㄱ

$\overrightarrow{\stackrel{\oplus}{\oplus}}$

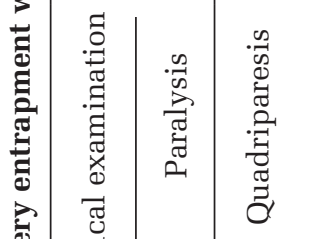

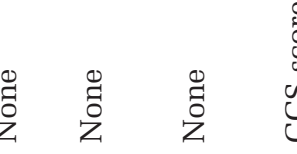

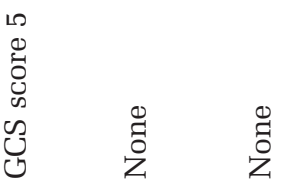

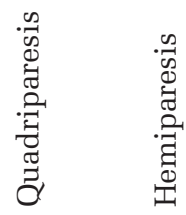<smiles>CCCCCC1CCCCC1</smiles> 


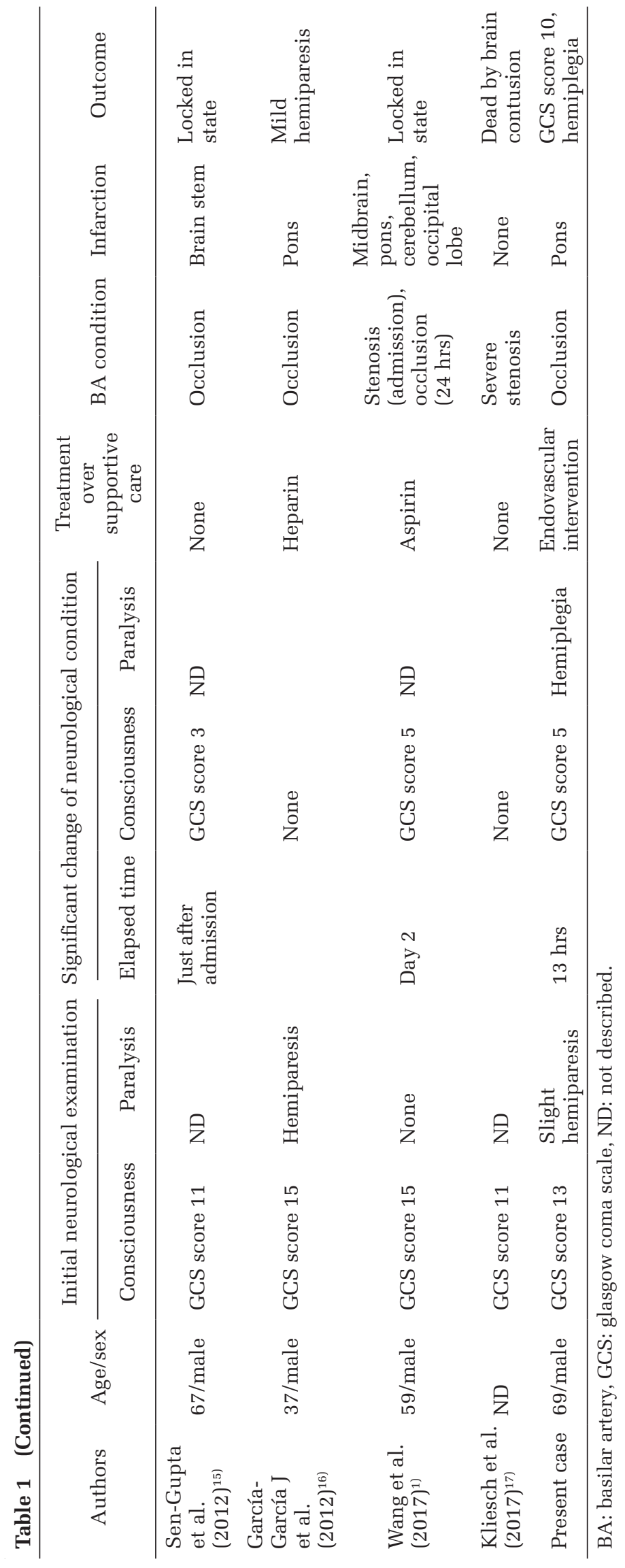

Neurol Med Chir (Tokyo) 58, August, 2018 


\section{References}

1) Wang A, Wainwright J, Cooper J, Tenner MS, Tandon A: Basilar artery herniation into the sphenoid sinus secondary to traumatic skull base fractures: case report and review of the literature. World Neurosurg 98: 878.e7-878.e10, 2017

2) Sato S, Iida H, Hirayama H, Endo M, Ohwada T, Fujii K: Traumatic basilar artery occlusion caused by a fracture of the clivus-case report. Neurol Med Chir (Tokyo) 41: 541-544, 2001

3) Cho J, Moon CT, Kang HS, et al.: Traumatic entrapment of the vertebrobasilar junction due to a longitudinal clival fracture: a case report. J Korean Med Sci 23: 747-751, 2008

4) Loop JW, White LE Jr, Shaw CM: Traumatic occlusion of the basilar artery within a clivus fracture. Radiology 83: 36-40, 1964

5) Lindenberg R: Incarceration of a vertebral artery in the cleft of a longitudinal fracture of the skull. Case report. J NeuroSurg 24: 908-910, 1966

6) Sights WP Jr: Incarceration of the basilar artery in a fracture of the clivus. Case report. J NeuroSurg 28: 588-591, 1968

7) Anthony DC, Atwater SK, Rozear MP, Burger PC: Occlusion of the basilar artery within a fracture of the clivus. Case report. J NeuroSurg 66: 929-931, 1987

8) Guha A, Fazl M, Cooper PW: Isolated basilar artery occlusion associated with a clivus fracture. Can J Neurol Sci 16: 81-83, 1989

9) Sato H, Sakai T, Uemura K: [A case of incarceration of the vertebral and basilar arteries in a longitudinal fracture of the clivus]. No Shinkei Geka 18: 1147-1150, 1990 (Japanese)

10) Taguchi $Y$, Matsuzawa $M$, Morishima $H$, Ono $H$, Oshima K, Hayakawa M: Incarceration of the basilar artery in a longitudinal fracture of the clivus: case report and literature review. J Trauma 48: 1148-1152, 2000

11) Bala A, Knuckey N, Wong G, Lee GY: Longitudinal clivus fracture associated with trapped basilar artery: unusual survival with good neurological recovery. J Clin Neurosci 11: 660-663, 2004

12) Kaakaji R, Russell EJ: Basilar artery herniation into the sphenoid sinus resulting in pontine and cerebellar infarction: demonstration by three-dimensional timeof-flight MR angiography. AJNR Am J Neuroradiol 25: 1348-1350, 2004
13) Khanna P, Bobinski M: Computed tomography and magnetic resonance imaging of a basilar artery herniation into the sphenoid sinus. Skull Base 20: 269-273, 2010

14) Fang J, Kuang L, Chen J, et al.: Posttraumatic basilar artery herniation associated with dissecting aneurysm formation: follow-up over 20 months. Cardiovasc Intervent Radiol 35: 1237-1241, 2012

15) Sen-Gupta I, Daiga DA, Alberts MJ: Teaching neuroimages: locked-in syndrome resulting from traumatic basilar artery occlusion following clivus fracture. Neurology 78: e148-e149, 2012

16) García-García J, Villar-Garcia M, Abad L, Segura T: Brainstem infarct due to traumatic basilar artery entrapment caused by longitudinal clival fracture. Arch Neurol 69: 662-663, 2012

17) Kliesch S, Bauknecht C, Bohner G, Liebig T, Siebert E: Traumatic basilar artery entrapment with patency of pontine perforators and absence of significant brainstem infarction: report of an unusual case. J Neurointerv Surg 9: e17, 2017

18) Hauck EF, Natarajan SK, Horvathy DB, Hopkins LN, Siddiqui AH, Levy EI: Stent-assisted basilar reconstruction for a traumatic vertebral dissection with a large basilar artery thrombosis. J Neurointerv Surg 3: 47-49, 2011

19) Li C, Li Y, Jiang C, Wu Z, Wang Y, Yang X: Stent alone treatment for dissections and dissecting aneurysms involving the basilar artery. J Neurointerv Surg 7: 50-55, 2015

20) Lee YY, Yoon W, Kim SK, et al.: Acute basilar artery occlusion: differences in characteristics and outcomes after endovascular therapy between patients with and without underlying severe atherosclerotic stenosis. AJNR Am J Neuroradiol 38: 1600-1604, 2017

21) Gerber JC, Daubner D, Kaiser D, et al.: Efficacy and safety of direct aspiration first pass technique versus stent-retriever thrombectomy in acute basilar artery occlusion-a retrospective single center experience. Neuroradiology 59: 297-304, 2017

Address reprint requests to: Fumiaki Kanamori, MD, Department of Neurosurgery, Tosei General Hospital, 160 Nishioiwake-cho, Seto, Aichi 489-8642, Japan. e-mail: shitetsu314@hotmail.co.jp 